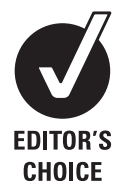

See Editorial, p 1461

'Department of Internal Medicine, University Hospital Basel, Basel, Switzerland ${ }^{2}$ Paris Descartes University, Department of Cardiology, Cochin Hospital, APHP, Paris, France

${ }^{3}$ Department of Cardiology, University Hospital Basel, Switzerland

${ }^{4}$ Department of Pneumology, Hospital del Mar-IMIM, UPF, CIBERES, ISC III, Barcelona,

Spain

${ }^{5}$ Emergengy Department, Hospital del Mar-IMIM, Barcelona, Spain

\section{Correspondence to}

Professor Christian Mueller, Department of Internal Medicine, University Hospital Basel, Petersgraben 4, CH-4031 Basel, Switzerland; chmueller@uhbs.ch

Accepted 3 March 2011 Published Online First 28 March 2011

\title{
The GRACE score's performance in predicting in-hospital and 1-year outcome in the era of high-sensitivity cardiac troponin assays and B-type natriuretic peptide
}

\author{
Christophe Meune, ${ }^{1,2}$ Beatrice Drexler, ${ }^{1}$ Philip Haaf, ${ }^{1}$ Tobias Reichlin,, \\ Miriam Reiter, ${ }^{1}$ Julia Meissner, ${ }^{1}$ Raphael Twerenbold, ${ }^{1}$ Claudia Stelzig, ${ }^{1}$ \\ Michael Freese, ${ }^{1}$ Katrin Winkler, ${ }^{4,5}$ Christian Mueller ${ }^{1,3}$
}

\begin{abstract}
Objective To compare the accuracy of the GRACE score, a strong prognosticator in acute coronary syndrome (ACS) that is calculated using conventional cardiac troponin (cTn) assays, with that calculated with highsensitivity cTn (hs-cTn) and with the combination of the GRACE score with hs-cTn or B-type natriuretic peptide (BNP).

Design Prospective international cohort.

Settings University Hospital.

Patients Patients enrolled in the Predictors of Acute Coronary Syndromes Evaluation prospective study with
\end{abstract} proven ACS.

Main outcome measured The capacity to predict in-hospital mortality, 1-year mortality and combined death/acute myocardial infarction (AMI) at 1 year.

Results 370 patients were enrolled (173 with unstable angina and 197 with AMI). In-hospital mortality was 4.1\%; 1-year mortality was $12.5 \%$. The GRACE score was significantly higher in patients who died than in those discharged alive (200 (174-222) vs 125 (98-155); $\mathrm{p}<0.001)$, and in those who died than in those who survived for 1 year (151 (133-169) vs 104 (85-125); $p<0.001)$. The area under the curve of the GRACE score was 0.87 regarding in-hospital mortality and 0.88 for 1-year mortality; if calculated with hs-cTn, it was 0.87 and 0.88 , respectively ( $p=N S$ for all comparisons). The addition of hs-cTn to the GRACE score resulted in no increased value, whereas the addition of BNP tended to improve 1-year mortality prediction $(p=0.058)$.

Conclusion The GRACE score is accurate for determining both in-hospital and long-term mortality in patients with ACS in the era of hs-cTn. The addition of hs-cTn or BNP to the GRACE score does not significantly improve risk prediction.

\section{INTRODUCTION}

Acute coronary syndrome (ACS) is a major health problem. ${ }^{12}$ As considerable variability exists among patients across the spectrum of ACS, accurate determination of risk has become a major focus in their initial evaluation. ${ }^{1-4}$ Numerous risk-prediction models for different types of patients with ACS exist, but the most widespread tool is the GRACE risk score. ${ }^{3-8}$ The GRACE score includes basic data from a large unselected population of patients with ACS, including those with ST-elevation acute myocardial infarction (STEMI), non-STEMI (NSTEMI) and unstable angina (UA). ${ }^{4}$ Initially designed to identify high-risk patients for in-hospital mortality, ${ }^{4}$ a second, simplified, model has been developed to predict 6-month mortality. ${ }^{5}$ The excellent ability of GRACE scores to discriminate the risk of death or combined death and acute myocardial infarction (AMI) has been confirmed for both 6 months ${ }^{9}$ and more prolonged periods up to 6 years. ${ }^{6}{ }^{10-12}$ Its use has recently been recommended by the European Society of Cardiology for risk stratification in patients with non-ST-elevation ACS. $^{2}$

Several biomarkers not considered in the previous scores have emerged as potential prognosticators in ACS, pointing to the need to re-evaluate scoring systems in the light of these new risk predictors. The recently developed high-sensitivity cardiac troponin (cTn) assays (hs-cTn) have greater accuracy than conventional assays in the early diagnosis of AMI. ${ }^{13}{ }^{14}$ However, concerns have been raised about possible increased hs-cTn levels in patients with a final diagnosis other than AMI and in lowrisk patients. ${ }^{15} 16$ Whether its incorporation into the calculation of the GRACE score will alter or improve the model and the effect of its addition to the GRACE score are not known.

The B-type natriuretic peptides (BNP and NT-proBNP) are strong prognosticators in ACS, independently of $\mathrm{cTn} .{ }^{17} 18$ The possible merit of their combination with the GRACE score varies among studies and their real effect remains unknown. ${ }^{11} 1920$ To resolve these issues, we compared the risk prediction conferred by the GRACE score using conventional cTn assay with hs-cTn assay for its calculation, and the combination of the GRACE score with hs-cTn or BNP measurements.

\section{METHODS}

\section{Patients}

The study population was derived from the Advantageous Predictors of Acute Coronary Syndromes Evaluation study, the first results of which have been reported. ${ }^{13}$ Briefly, the Advantageous Predictors of Acute Coronary Syndromes Evaluation is an ongoing prospective, international, multicentre study, designed and coordinated by the University Hospital Basel, which enrolled all patients with suspected AMI of less than $12 \mathrm{~h}$. 
The present study analysed the subgroup of patients with a final confirmed diagnosis of ACS, covering its full spectrum, from UA to NSTEMI and STEMI. The final diagnosis of ACS was based on all clinical, laboratory (but not hs-cTn and BNP concentrations) and imaging data available and was separately confirmed by two cardiologists. Disagreements between the observers were settled by a third cardiologist. AMI was defined according to the universal definition and required the association of clinical signs of myocardial ischaemia with the observation of a rising or falling pattern of local cTn concentration; when the 99th centile of the test could not be measured with the precision required, the $10 \%$ coefficient variation (CV) level was used as the cut-off value. $^{21} 22$ UA was diagnosed in the presence of (1) clinical manifestations suggesting myocardial ischaemia, without evidence of myocardial necrosis and (2) an ECG indicating ongoing ischaemia, or a $>70 \%$ stenosis of an epicardial coronary artery on coronary angiography, or a positive result on a cardiac exercise test, or when the diagnosis was uncertain but follow-up information showed that the patient suffered an AMI or sudden unexpected cardiac death within 60 days.

The ethics committee at each institution approved this study, and all patients gave written informed consent to participate.

\section{Measurement of biomarkers}

Blood samples were collected in EDTA tubes at admission and centrifuged at $4^{\circ} \mathrm{C}$ within $15 \mathrm{~min}$ of collection. Conventional cTn assays were immediately analysed, and the results were available to the emergency department practitioner. The following cTn assays were used for the clinical care of the patients at the participating hospitals: Abbott Axsym cTnI ADV (limit of detection $0.02 \mu \mathrm{g} / \mathrm{l}$; 99th centile cut-off point $<0.08 \mu \mathrm{g} / \mathrm{l}$; CV $\leq 10 \%$ for $0.16 \mu \mathrm{g} / \mathrm{l})$, Beckmann Coulter Accu cTnI (limit of detection $0.01 \mu \mathrm{g} / 1$; 99th centile cut-off point $<0.04 \mu \mathrm{g} / \mathrm{l} ; \mathrm{CV} \leq 10 \%$ for $0.06 \mu \mathrm{g} / \mathrm{l}$ ) and Roche cTn 4th generation (limit of detection of $0.01 \mu \mathrm{g} / \mathrm{l}$; 99th centile cut-off point $<0.01 \mu \mathrm{g} / \mathrm{l} ; \mathrm{CV} \leq 10 \%$ for $0.035 \mu \mathrm{g} / \mathrm{l}$ ). Plasma hs-cTn concentrations were measured using a commercially available electrochemiluminescence immunoassay (hs-cTn, Roche Diagnostics, Mannheim, Germany). The 99th centile with a CV $\leq 10 \%$ is achieved for $14 \mathrm{ng} / \mathrm{l}^{23} \mathrm{BNP}$ was measured using the commercially available Biosite Diagnostics assay (Biosite Diagnostics, La Jolla, California, USA); its analytical sensitivity is $<5.0 \mathrm{pg} /$ $\mathrm{ml}$, with a measurable range of $0-5000 \mathrm{pg} / \mathrm{ml}$.

\section{Calculation of GRACE score}

For in-hospital prediction, the GRACE score was calculated on the basis of admission characteristics using the followings items: Killip class, systolic blood pressure, heart rate, age, creatinine concentration, cardiac arrest, the presence of any ST-segment deviation, the presence/absence of elevated cardiac markers. ${ }^{4}$

For long-term prediction, the simplified GRACE model was calculated on the basis of admission characteristics using the following items: age, previous heart failure, past AMI, heart rate, systolic blood pressure, the presence of ST-segment depression, creatinine concentration, elevated cardiac markers, no in-hospital percutaneous coronary intervention. ${ }^{5}$

For the present analysis, a single value from the conventional assay of cTn above the cut-off value was determined as a score for elevated biomarkers of myocardial necrosis.

\section{Studied end points}

We measured the capacity of the GRACE score to predict in-hospital death, 1-year mortality and combined death/AMI at 1 year. We chose to evaluate the end points at these two time points because they represent short- and long-term follow-up periods, respectively. In addition, the prognostic significance of both the GRACE score and BNP has been demonstrated at 1 year. $^{12} 17$

\section{Statistical analysis}

The data are expressed as means \pm SD for Gaussian continuous variables, median (IOR) for non-Gaussian continuous variables, and numbers and percentages for categorical variables. The baseline characteristics were analysed using the $\chi^{2}$ test or Fisher exact test and the Student t test or Mann-Whitney test, as appropriate, for comparison between groups defined by the presence or absence of the end points. The existence of a correlation between BNP, hs-cTn and the GRACE risk score was investigated using the Spearman correlation test. The possible role played by variables in relation to the end points was examined first in a single-variable analysis, followed by logistic regression. We then compared the predictive accuracy of the GRACE score with its recalculation with hs-cTn, the combined GRACE+hs-cTn, GRACE+BNP using receiver operating characteristics curves, and integrated discrimination improvement (IDI) (figure 1). ${ }^{24} 25 \mathrm{p}<0.05$ was considered significant. Stata statistical software V.10.1 was used for all analyses.

\section{RESULTS}

The study population consisted of 370 patients comprising 50 with STEMI, 147 with non-STEMI, and 173 with UA. Using hs-cTn rather than the conventional assay of cTn, in conjunction with the universal definition for the confirmed diagnosis of AMI, would have led to reclassification of 28 patients from UA to AMI. ${ }^{21}$ The GRACE score shifted from $120(102-148)$ to 134 $(109-156)$ in these patients $(p<0.267)$. No patients were lost to follow-up. Follow-up was 100\% complete at discharge from hospital and $84.3 \%$ complete at 1 year. The detailed characteristics of the study group, including comparison of the survivors and non-survivors, are shown in table 1.

\section{In-hospital prognosis}

The GRACE score for predicting in-hospital outcome was 126 (99-159) in the entire cohort, ranging from149 (123-185) in patients with STEMI to $123(96-155)$ in patients with UA/ NSTEMI. Fifteen patients $(4.1 \%)$ died during hospitalisation. Compared with the patients who were discharged alive, the patients who died during initial hospitalisation were older and
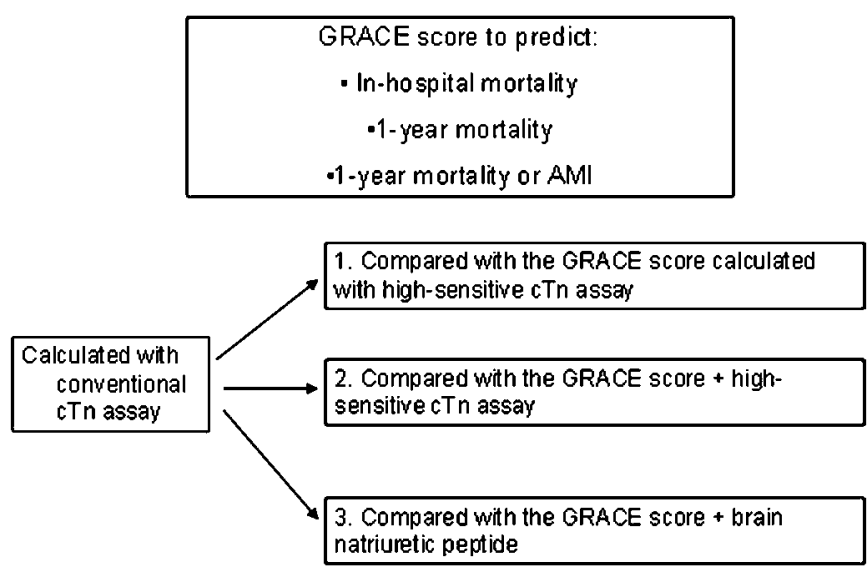

Figure 1 Different comparisons performed. AMI, acute myocardial infarction; cTn, cardiac troponin. 
Table 1 Baseline characteristics

\begin{tabular}{|c|c|c|c|}
\hline & Total $(n=370)$ & $\begin{array}{l}\text { Death in hospital } \\
(n=15)\end{array}$ & $\begin{array}{l}\text { Death at } 1 \\
\text { year }(n=39)\end{array}$ \\
\hline Age (years) & $71(59-78)$ & $76(75-86)^{*}$ & $84(75-89)^{*}$ \\
\hline Male sex & $273(73.8)$ & $11(73.3)$ & $27(69.2)$ \\
\hline Hypertension & $293(79.2)$ & $14(93.3)$ & $34(87.2)$ \\
\hline Hypercholesterolaemia & $223(60.3)$ & $7(46.7)$ & $19(48.7)$ \\
\hline Diabetes & $102(27.6)$ & $6(40.0)$ & $15(38.5)$ \\
\hline Body mass index $\left(\mathrm{kg} / \mathrm{m}^{2}\right)$ & $27.0 \pm 4.4$ & $25.7 \pm 4.2$ & $25.3 \pm 4.1^{*}$ \\
\hline Family history (1st degree) & 68 (38.9) & $3(50.0)$ & $3(33.3)$ \\
\hline Smoking & $89(24.2)$ & $4(26.7)$ & $11(28.2)$ \\
\hline Risk factors $\geq 2$ & $272(73.5)$ & $11(73.3)$ & $26(66.7)$ \\
\hline Past myocardial infarction & $148(40.0)$ & $4(26.7)$ & $20(51.3)$ \\
\hline Past coronary artery disease & $209(56.5)$ & $9(60.0)$ & $29(74.4)^{*}$ \\
\hline Known renal failure & $58(15.7)$ & $5(33.3)$ & $19(48.7)^{*}$ \\
\hline \multicolumn{4}{|l|}{ Drug regimen } \\
\hline Aspirin & $213(57.6)$ & $8(53.3)$ & $21(53.9)$ \\
\hline Clopidogrel & $64(17.3)$ & $0(0)$ & $7(18.0)$ \\
\hline$\beta$-adrenergic blocker & $187(50.5)$ & $6(40.0)$ & $24(61.5)$ \\
\hline ACE inhibitor/ARB & $200(54.1)$ & $11(73.3)$ & $25(64.1)$ \\
\hline Statin & $188(50.8)$ & $6(40.0)$ & $22(56.4)$ \\
\hline Duration of chest pain (h) & $4.5 \pm 3.2$ & $4.4 \pm 2.7$ & $5.0 \pm 3.8$ \\
\hline \multicolumn{4}{|l|}{ Clinical presentation } \\
\hline Heart rate on admission (beats/min) & $77 \pm 18$ & $91 \pm 33^{*}$ & $89 \pm 25^{*}$ \\
\hline Systolic BP (mm Hg) & $145 \pm 26$ & $140 \pm 36$ & $134 \pm 33^{*}$ \\
\hline Diastolic BP (mm Hg) & $83 \pm 15$ & $85 \pm 22$ & $78 \pm 21^{*}$ \\
\hline ST segment deviation & $151(40.8)$ & $11(73.3)^{*}$ & $33(84.6)^{*}$ \\
\hline \multicolumn{4}{|l|}{ Screening blood tests } \\
\hline Creatinine $(\mu \mathrm{mol} / \mathrm{l})$ & $91 \pm 46$ & $108 \pm 41$ & $118 \pm 48^{*}$ \\
\hline GFR $\left(\mathrm{ml} / \mathrm{min} / 1.73 \mathrm{~m}^{2}\right)$ & $82 \pm 27$ & $65 \pm 21 *$ & $60 \pm 26^{*}$ \\
\hline Haemoglobin (g/dl) & $140 \pm 18$ & $139 \pm 15$ & $125 \pm 23$ \\
\hline cTn $>99$ th centile & $192(77.7)$ & $7(47.7)$ & $26(66.7)^{*}$ \\
\hline hs-cTn (ng/l) & $28(10-121)$ & $78(27-224)^{*}$ & $147(30-470)^{*}$ \\
\hline hs-cTn $>99$ th centile & $237(67.9)$ & $14(93.3)^{*}$ & $36(92.3)^{*}$ \\
\hline BNP (pg/ml) & $139(64-369)$ & $487(229-885)^{*}$ & $558(322-1183)^{*}$ \\
\hline Coronary angiography & $250(67.6)$ & $8(53.3)$ & $16(41.0)^{*}$ \\
\hline $\mathrm{PCl}$ & $177(47.8)$ & $4(26.7)$ & $10(25.6)^{*}$ \\
\hline Coronary artery bypass grafting & $34(9.2)$ & $3(20.0)$ & $3(7.7)$ \\
\hline
\end{tabular}

had a higher heart rate and reduced glomerular filtration rate at admission (table 1). In addition, they had increased hs-cTn (78 $(28-224)$ vs $27(10-119) \mathrm{ng} / \mathrm{l}$, respectively; $\mathrm{p}=0.015)$ and BNP (487 (229-885) vs 135 (64-338) pg/ml; p=0.005).

There was a significant but only partial correlation between the GRACE risk score and hs-cTn concentrations ( $\mathrm{r}=0.54$, $\mathrm{p}<0.001)$ or BNP $(\mathrm{r}=0.62, \mathrm{p}<0.001)$. The GRACE score was increased in patients who died versus those discharged alive (200 $(174-222)$ vs 125 (98-155); $\mathrm{p}<0.001)$. The GRACE score is the only variable that remains associated with in-hospital mortality according to multivariate analysis $(\mathrm{p}<0.001)$. Table 2 presents the predictive accuracy of the different models. The consideration of increased hs-cTn (above the 99th centile) rather than increased conventional assay of cTn for the calculation of the GRACE score did not alter the model. The addition of neither hs-cTn concentration nor BNP concentration to the GRACE score improved the model (table 2).

\section{One-year follow-up}

The patients mostly had a low (score <90, 27.5\%) or intermediate (score from 90 to $120,35.8 \%$ ) risk of 1 -year death as assessed by the GRACE score. Thirty-nine (12.5\%) patients died during the 1-year follow-up period, and 56 reached the combined end point of death/recurrence of AMI (18.0\%). The variables that are associated with 1-year mortality are reported in table 1. Patients who died had higher hs-cTn concentrations (147 (30-470) vs $23(9-103) \mathrm{ng} / \mathrm{l} ; \mathrm{p}<0.001)$, BNP concentrations (558 (322-1183) vs $120(58-249) \mathrm{pg} / \mathrm{ml} ; \mathrm{p}<0.001)$ and GRACE score $(151(133-169)$ vs $104(85-125) ; p<0.001)$ than those who survived. The GRACE risk score $(p<0.001)$ and the presence of ST-segment deviation ( $\mathrm{p}=0.002)$, in-hospital revascularisation $(p=0.011)$ and glomerular filtration rate $(p=0.022)$ remained associated with 1-year mortality in multivariate analysis. The GRACE score accurately predicted the 1-year mortality end point. Its calculation using increased hs-cTn did not alter the model (table 2). The addition of hs-cTn to the GRACE score did not improve the model. There was a trend for an improvement conferred by combining BNP concentrations with the score, as expressed by the IDI but not c-statistics (table 2).

Lastly, the GRACE risk score predicts correctly, but less adequately, the composite end point of death/recurrence of AMI at 1 year. The model was neither altered by the consideration of increased hs-cTn nor improved by the addition of hs-cTn or BNP consideration to the risk score (table 2). 
Table 2 Accuracy of risk prediction of the different models

\begin{tabular}{|c|c|c|c|c|}
\hline & \multicolumn{2}{|l|}{ C-statistics } & \multicolumn{2}{|l|}{ IDI } \\
\hline & $\overline{A U C}$ & p Value* & IDI value & p Value* \\
\hline \multicolumn{5}{|l|}{ In-hospital mortality } \\
\hline GRACE score & $0.87(0.75-0.99)$ & - & Reference & - \\
\hline GRACE score using hs-cTn & $0.87(0.77-0.98)$ & 0.840 & 0.44 & 0.662 \\
\hline GRACE score + hs-cTn concentration & $0.87(0.75-0.99)$ & 0.966 & 0.59 & 0.555 \\
\hline GRACE score + BNP concentration & $0.88(0.78-0.99)$ & 0.583 & 0.15 & 0.877 \\
\hline \multicolumn{5}{|l|}{ One-year mortality $\dagger$} \\
\hline GRACE score & $0.88(0.81-0.95)$ & - & Reference & - \\
\hline GRACE score using hs-cTn & $0.88(0.81-0.95)$ & 0.814 & 0.26 & 0.797 \\
\hline GRACE score + hs-cTn concentration & $0.88(0.81-0.95)$ & 0.640 & $<0.01$ & 0.999 \\
\hline GRACE score + BNP concentration & $0.88(0.81-0.95)$ & 0.322 & 1.90 & 0.058 \\
\hline \multicolumn{5}{|l|}{ One-year combined of death/AMI† } \\
\hline GRACE score & $0.75(0.66-0.84)$ & - & Reference & - \\
\hline GRACE score using hs-cTn & $0.76(0.67-0.85)$ & 0.669 & 0.35 & 0.726 \\
\hline GRACE score + hs-cTn concentration & $0.75(0.66-0.84)$ & 0.740 & 0.35 & 0.724 \\
\hline GRACE score + BNP concentration & $0.75(0.67-0.84)$ & 0.978 & 1.25 & 0.210 \\
\hline
\end{tabular}

\section{DISCUSSION}

We made clinically important observations in this study. First, the GRACE risk score accurately predicted both in-hospital and 1-year mortality, and less accurately the combined 1-year death and AMI. Second, the use of increased hs-cTn instead of conventional cTn in the calculation of the GRACE risk score did not alter the model. Third, the combination of hs-cTn or BNP concentrations with the GRACE score had no significant incremental value.

The in-hospital and 1-year prognostic values of the GRACE score were excellent in our study, and we observed even higher AUC than in the validation sets of the GRACE registries. ${ }^{4} 59$ Such findings were expected and emphasise that a prediction tool is as generalisable as the population from which it is derived. The GRACE registry is a multinational registry involving 94 hospitals in 14 countries; it includes more than 10000 patients and was designed for prognosis of all-cause mortality. Its accuracy in the prediction of mortality has been confirmed in several studies at different time points. ${ }^{6} 10{ }^{12}$ In addition, the GRACE score predicted fairly, but less accurately, combined 1-year death/AMI, a result that is consistent with previous studies. ${ }^{9}{ }^{20}$ More importantly, we observe that the use of increased hs-cTn rather than conventional cTn for its calculation does not alter the model.

Over the last few decades, cTn has emerged as the key biomarker for detecting ACS in patients with chest pain-related symptoms. ${ }^{26} 27$ Recently, high-sensitivity assays of cTn have demonstrated their superiority over conventional assays for detecting AMI and overall ACS. ${ }^{13} 1428$ They are scheduled to soon become the only available cTn assays. Although some authors have reported a possible specificity deficit of these new assays, ${ }^{15} 16$ our study demonstrates that the GRACE risk calculation is still valid with the use of these new assays. This may have important clinical implications. Initial risk stratification of patients with ACS is crucial for making appropriate decisions about the need for transfer to a tertiary centre. In addition, patients at highest risk may derive greater benefits from intense pharmacological treatment and interventions, the benefits of which may outweigh the risk of adverse effects. ${ }^{1} 2$

BNP is a marker of cardiac stress and its accuracy as a strong prognosticator has been proven even in the absence of left ventricular dysfunction and independently of certain elements of the GRACE score such as age, ST deviation and cTn concentration. ${ }^{17} 1829$ However, there are conflicting results on whether the addition of BNP concentration to the GRACE score improves risk stratification. Beygui and colleagues demonstrated in a retrospective analysis of the ARCHIPELAGO study (irbesartan in patients with ACS without ST segment elevation) that the performance of the GRACE score was significantly improved by the introduction of BNP concentration to predict death/heart failure but not the composite end point of death, stroke, AMI, recurrent ischaemia or unplanned hospitalisation. ${ }^{20}$ In addition, this study investigated patients with long-lasting symptoms (up to $48 \mathrm{~h}$ ) at low risk of cardiac events (2-month mortality of $0.7 \%$ ). In another study, Ang and colleagues reported that BNP concentration did not improve the risk prediction offered by the GRACE score, as expressed by the c-statistics. ${ }^{19}$ Our results are consistent with these studies, as we report no significant difference in the c-statistics or the IDI offered by the addition of BNP concentrations to the GRACE score.

In this study, we examined patients across the entire spectrum of ACS and focused on mortality because the GRACE score was developed and validated mainly for this indication. We demonstrate that neither BNP nor hs-cTn added significant value to the GRACE score. Subgroup analysis showed no difference in in-hospital mortality prediction $(p=0.497$ and $p=0.930$, respectively) and 1 -year mortality ( $p=0.802$ and $p=0.860$, respectively) of the different models in patients with STEMI and UA/NSTEMI, respectively. The possible merit of using inflammatory markers, structural enzymes, markers of platelet activation and neurohormones in addition to the GRACE score has been previously investigated. Only interleukin-6 demonstrated incremental value. ${ }^{11} 1920$ These previous results and ours should not be considered to be a contradiction of the prognostic significance of either hs-cTn or BNP. As we report a very high AUC $(>0.85)$ in all our analyses, our results should rather be interpreted as the high capacity to discriminate patients at high risk offered by the GRACE score.

Receiver operating characteristics analysis is designed ideally to measure the accuracy of a single predictor across its broad concentration. Such analysis can also easily compare two single predictors in a head-to-head fashion, but is not ideal for 
comparing a combination of predictors with a single predictor. In addition, it may lack sensitivity. Recently, the net reclassification improvement and IDI have emerged as the preferred methods for investigating the predictive ability of new biomarkers or new risk scores. ${ }^{25}$ In the present study, we compared the predictive ability of the different models using both c-statistics and the IDI; we assume therefore that our results are robust.

Our study should be interpreted within its limitations. First, patients with severe renal failure were excluded from the study. Therefore, no conclusion can be drawn about this specific population. Second, the sample size is relatively small compared with the registries that were used to create and validate the GRACE score. ${ }^{4}$ However, we found a similar accuracy of the GRACE score to that reported in these studies, which may suggest that our population is an adequate representation of the spectrum of patients with ACS. In our study, the rate of in-hospital percutaneous coronary intervention ranged from $45 \%$ for UA to $80 \%$ for STEMI. As cardiac markers are related to the extent of coronary disease and possible revascularisation, cTn and hs-cTn may have lost their prognostic effect after successful revascularisation. Other components of the GRACE score maintain prognostic relevance, explaining the absence of difference that we observed in the prognostic significance of the GRACE score using different cardiac markers for its calculation.

\section{CONCLUSION}

The GRACE risk prediction score is still accurate for determining both in-hospital and long-term mortality in patients with ACS in the era of high-sensitivity assays of cardiac troponin. The addition of hs-cTn or BNP to the GRACE score does not improve the risk prediction offered by the GRACE score alone.

Funding This study was supported by grants from the Swiss National Science Foundation (PP00B-102853), the Swiss Heart Foundation, Abbott, Roche, Siemens and the Department of Internal Medicine, University Hospital Basel. CM was supported by a grant from the Freie Akademische Gesellschaft Basel (FAG).

Competing interests $\mathrm{CM}$ received research grant support from Abbott, Brahms, nanosphere, Roche and Siemens, consulting fees from Abbott, and lecture fees from Abbott, Biosite, Brahms, Roche and Siemens.

Patient consent Obtained.

Ethics approval This study was conducted with the approval of the University Hospital Basel.

Provenance and peer review Not commissioned; externally peer reviewed.

\section{REFERENCES}

1. Anderson JL, Adams CD, Antman EM, et al. ACC/AHA 2007 guidelines for the management of patients with unstable angina/non ST-elevation myocardial infarction: a report of the American College of Cardiology/American Heart Association Task Force on Practice Guidelines (Writing Committee to Revise the 2002 Guidelines for the Management of Patients With Unstable Angina/Non ST-Elevation Myocardial Infarction): developed in collaboration with the American College of Emergency Physicians, the Society for Cardiovascular Angiography and Interventions, and the Society of Thoracic Surgeons: endorsed by the American Association of Cardiovascular and Pulmonary Rehabilitation and the Society for Academic Emergency Medicine. Circulation 2007;116:e148-304.

2. Bassand JP, Hamm CW, Ardissino D, et al. Guidelines for the diagnosis and treatment of non-ST-segment elevation acute coronary syndromes. Eur Heart $J$ 2007:28:1598-660.

3. Antman EM, Cohen M, Bernink PJ, et al. The TIMI risk score for unstable angina/ non-ST elevation Ml: A method for prognostication and therapeutic decision making. JAMA 2000;284:835-42.
4. Granger CB, Goldberg RJ, Dabbous 0, et al. Predictors of hospital mortality in the global registry of acute coronary events. Arch Intern Med 2003;163:2345-53.

5. Eagle KA, Lim MJ, Dabbous $\mathrm{OH}$, et al. A validated prediction model for all forms of acute coronary syndrome: estimating the risk of 6-month postdischarge death in an international registry. JAMA 2004;291:2727-33.

6. Kozieradzka A, Kaminski KA, Maciorkowska D, et al. GRACE, TIMI, Zwolle and CADILLAC risk scores-Do they predict 5-year outcomes after ST-elevation myocardia infarction treated invasively? Int J Cardiol 2011;148:70-5

7. Lindahl B, Toss $\mathrm{H}$, Siegbahn A, et al. Markers of myocardial damage and inflammation in relation to long-term mortality in unstable coronary artery disease. FRISC Study Group. Fragmin during Instability in Coronary Artery Disease. N Eng/ Med 2000;343:1139-47.

8. Morrow DA, Antman EM, Giugliano RP, et al. A simple risk index for rapid initial triage of patients with ST-elevation myocardial infarction: an InTIME II substudy. Lancet 2001:358:1571-5.

9. Fox KA, Dabbous OH, Goldberg RJ, et al. Prediction of risk of death and myocardia infarction in the six months after presentation with acute coronary syndrome: prospective multinational observational study (GRACE). BMJ 2006;333:1091.

10. de Araujo Goncalves P, Ferreira J, Aguiar C, et al. PURSUIT, and GRACE risk scores: sustained prognostic value and interaction with revascularization in NSTE-ACS. Eur Heart J 2005;26:865-72.

11. Eggers KM, Kempf $T$, Venge $P$, et al. Improving long-term risk prediction in patients with acute chest pain: the Global Registry of Acute Coronary Events (GRACE) risk score is enhanced by selected nonnecrosis biomarkers. Am Heart $J$ 2010:160:88-94

12. Yan AT, Yan RT, Tan M, et al. Risk scores for risk stratification in acute coronary syndromes: useful but simpler is not necessarily better. Eur Heart $J$ 2007:28:1072-8.

13. Reichlin T, Hochholzer W, Bassetti S, et al. Early diagnosis of myocardial infarction with sensitive cardiac troponin assays. N Engl J Med 2009;361:858-67.

14. Keller T, Zeller T, Peetz D, et al. Sensitive troponin I assay in early diagnosis of acute myocardial infarction. N Engl J Med 2009;361:868-77.

15. Neizel M, Steen H, Korosoglou G, et al. Minor troponin T elevation in patients 6 months after acute myocardial infarction: an observational study. Clin Res Cardiol 2009;98:297-304.

16. Omland T, de Lemos JA, Sabatine MS, et al. A sensitive cardiac troponin T assay in stable coronary artery disease. N Engl J Med 2009:361:2538-47.

17. de Lemos JA, Morrow DA, Bentley $\mathbf{J H}$, et al. The prognostic value of B-type natriuretic peptide in patients with acute coronary syndromes. $N$ Engl J Med 2001;345:1014-21.

18. Jernberg T, Stridsberg M, Venge $\mathrm{P}$, et al. N-terminal pro brain natriuretic peptide on admission for early risk stratification of patients with chest pain and no ST-segment elevation. J Am Coll Cardiol 2002;40:437-45.

19. Ang DS, Wei L, Kao MP, et al. A comparison between B-type natriuretic peptide, global registry of acute coronary events (GRACE) score and their combination in ACS risk stratification. Heart 2009;95:1836-42.

20. Beygui F, Silvain J, Pena A, et al. Usefulness of biomarker strategy to improve GRACE score's prediction performance in patients with non-ST-segment elevation acute coronary syndrome and low event rates. Am J Cardiol 2010;106:650-8.

21. Thygesen K, Alpert JS, White HD, et al. Universal definition of myocardial infarction. Circulation 2007;116:2634-53

22. Apple FS, Jesse RL, Newby LK, et al. National Academy of Clinical Biochemistry and IFCC Committee for Standardization of Markers of Cardiac Damage Laboratory Medicine Practice Guidelines: Analytical issues for biochemical markers of acute coronary syndromes. Circulation 2007;115:e352-5

23. Giannitsis $\mathbf{E}$, Kurz $\mathrm{K}$, Hallermayer $\mathrm{K}$, et al. Analytical validation of a high-sensitivity cardiac troponin T assay. Clin Chem 2010;56:254-61.

24. DeLong ER, DeLong DM, Clarke-Pearson DL. Comparing the areas under two or more correlated receiver operating characteristic curves: a nonparametric approach. Biometrics 1988;44:837-45.

25. Pencina MJ, D'Agostino RB Sr, D'Agostino RB Jr, et al. Evaluating the added predictive ability of a new marker: from area under the ROC curve to reclassification and beyond. Stat Med 2008;27:157-72; discussion 207-12.

26. Katus HA, Remppis A, Neumann FJ, et al. Diagnostic efficiency of troponin $T$ measurements in acute myocardial infarction. Circulation 1991;83:902-12.

27. Morrow DA, Cannon CP, Jesse RL, et al. National Academy of Clinical Biochemistry Laboratory Medicine Practice Guidelines: Clinical characteristics and utilization of biochemical markers in acute coronary syndromes. Circulation 2007;115:e356-75.

28. Giannitsis $\mathbf{E}$, Becker $\mathrm{M}, \mathrm{Kurz} \mathrm{K}$, et al. High-sensitivity cardiac troponin T for early prediction of evolving non-ST-segment elevation myocardial infarction in patients with suspected acute coronary syndrome and negative troponin results on admission. Clin Chem 2010:56:642-50.

29. Mega JL, Morrow DA, De Lemos JA, et al. B-type natriuretic peptide at presentation and prognosis in patients with ST-segment elevation myocardial infarction: an ENTIRE-TIMI-23 substudy. J Am Coll Cardiol 2004;44:335-9. 